REVIEW

\title{
Developmental delay: when to suspect and how to investigate for an inborn error of metabolism
}

\author{
M A Cleary, A Green
}

Arch Dis Child 2005;90:1128-1132. doi: 10.1136/adc.2005.072025

The purpose of this review is to provide a practical guideline on the suspicion and investigation of inborn errors of metabolism (IEMs) as cause of developmental delay. Developmental delay is a common paediatric problem. Inborn errors of metabolism are a rare cause of developmental delay. However, it is important to detect IEMs for several reasons: accurate counselling may be given regarding recurrence risk; metabolic decompensation may be avoided; and specific treatments may be available. Certain clinical situations are more likely to point to an IEM as the cause of developmental delay. This review highlights the risk factors in the history, the important examination findings, and the appropriate biochemical investigation of the child with developmental delay. Following these guidelines makes "missing" an IEM unlikely.

See end of article for authors' affiliations

\section{Correspondence to:} Dr M A Cleary, Dept of Metabolic Medicine, Great Ormond St Hospital for Sick Children, London WCIN 3JH, UKK; ClearM@ gosh.nhs.uk

Accepted 19 August 2005
D evelopmental disabilities occur in approximately $5-10 \%$ of the childhood population. ${ }^{1}$ Global developmental delay is described as occurring when there is significant delay in two or more developmental domains (gross motor, fine motor, cognition, speech/ language, and personal/social). The term global developmental delay is defined as performance at least two standard deviations below the mean of age appropriate, standardised norm referenced developmental testing. ${ }^{1}$ This term is used for younger children (typically less than 5 years), whereas the term mental retardation is used for older children where IQ testing is more reliable. Global delay prevalence is estimated to be $1-3 \%$ of children aged less than 5 years. ${ }^{1}$

Identification of developmental delay occurs in countries with well developed systems of health care through surveillance programmes but may be recognised by parents, the family doctor, or other health practitioners. Once recognised, developmentally delayed children are frequently referred to a paediatrician; more commonly community based but in some areas hospital clinics. This assessment will confirm the existence of delay, characterise the domains affected, and attempt to establish the aetiology. After history and examination are complete, confirmation of aetiology of the developmental delay may be possible in approximately one fifth of children. ${ }^{2}$ Thereafter laboratory investigations and other investigative approaches are considered, such as magnetic resonance imaging of the brain. The commoner causes of developmental delay are chromosomal abnormalities and structural brain abnormalities. ${ }^{2}$ One of the challenging questions for paediatricians investigating children with developmental delay is how intensively should one investigate for inborn errors of metabolism and which metabolic investigations are indicated.

Reports on the prevalence of inborn errors of metabolism (IEMs) as the underlying cause of delay show some variation but generally indicate that IEMs are found to be the cause in only a small percentage of these cases ${ }^{3}$ (that is, 1-5\%). In fact the American College of Medical Genetics in 1997 recommended omitting metabolic screening in unselected cases of developmental delay. ${ }^{3}$ The report from the American Academy of Neurology and the Practice Committee of the Child Neurology Society offers guidelines based on a review of published literature. "Screening" metabolic investigations are recommended especially where newborn screening is absent or minimal, and directed metabolic tests are advocated thereafter based on clinical findings. ${ }^{1}$

The incidence and range of IEM as a cause of developmental delay is likely to vary between different populations; of note the more recent reported series are from the USA. ${ }^{13}$ There is a paucity of data on the detection of metabolic disorders when investigating developmental delay in the United Kingdom. A report from 1998 identified the wide variation both in the number and type of investigations chosen by a group of UK community paediatricians when investigating developmental delay. ${ }^{4}$ Unpublished data of a retrospective analysis (1995-2001) of requests for IEM investigations to the Clinical Biochemistry Department at Birmingham Children's Hospital diagnosed 40 cases of IEM over a six year period. The commonest causes were disorders of organic and amino acid metabolism and the mucopolysaccharidoses. This was not a systematic study and therefore we do not know how many total cases of developmental delay were diagnosed in that period; no conclusions about prevalence of IEM in this population can therefore be drawn. However, the types and range of disorders found are probably representative of other larger UK conurbations with a similar ethnic mix. Another interesting feature of these diagnoses was the finding of unrelated disorders-that is, where the biochemical defect was not a cause of the developmental delay; for example, cystinuria and other biochemical abnormalities with no associated clinical effects, e.g. prolinaemia, sarcosinaemia. 
Table 1 Important history details suggestive of IEM

\begin{tabular}{ll}
\hline & Features to suggest an IEM \\
\hline Pregnancy & Maternal health \\
& HELLP, AFLP \\
Family history & Consanguinity \\
& Unexplained neonatal or infantile \\
& deaths \\
& Three generation pedigree \\
Past medical history of delayed & Unexplained hypoglycaemia \\
child & Encephalopathy \\
& Protein aversion \\
& Self-injurious behaviour \\
& Psychiatric symptoms \\
& Seizure disorder \\
Type of developmental delay & Regression \\
& Hypotonia \\
& Speech delay \\
\hline
\end{tabular}

IEM are rare causes of isolated developmental delay. More usually, an IEM occurs in association with other clinical clues. However, despite their relatively low incidence, IEMs remain extremely important causes to determine since specific therapies may be available for the condition, acute metabolic decompensation may be avoidable, and accurate prognosis and genetic counselling can be offered. Unlike other parts of the world, in the UK, universal newborn screening is currently restricted to phenylketonuria and congenital hypothyroidism (CHT); other IEMs, for example, maple syrup urine disease, urea cycle defects, and organic acid disorders will not therefore be diagnosed by this route. In some countries newborn screening by tandem mass spectrometry will detect organic acid disorders, and some amino acid and urea cycle disorders. ${ }^{36}$ It is particularly important, therefore, to investigate infants/children with developmental delay where extended newborn screening for metabolic disorders is not provided. Although the newborn screening programme in the UK should detect primary CHT, patients with secondary or acquired late onset forms will not be diagnosed.

This article describes an investigative approach to the child with developmental delay focusing on the appropriate testing for IEMs. It is not an evidence based approach due to the lack of available UK data; it is a practical guide to investigating children with developmental delay based on personal practice from two major paediatric centres within the UK.

\section{CLUES FROM HISTORY AND EXAMINATION}

In the history there may be several important features to suggest an IEM (table 1). The previous family history should be explored for evidence of consanguinity and unexplained neonatal or infantile deaths; this should include a three generation pedigree. The pregnancy and birth details should be noted. Children with IEMs are usually born of normal birth weight and in good condition. Exceptions to this are those disorders which have intra-uterine or immediate effects following birth, such as non-ketotic hyperglycinaemia and disorders of pyruvate metabolism. A history of maternal acute fatty liver of pregnancy (AFLP) or haemolysis elevated liver enzymes and low platelets (HELLP) is associated with several fatty acid oxidation disorders.

The past medical history of the developmentally delayed child should be questioned in detail. Useful clues to an underlying IEM are a history of hypoglycaemia, an episode of unexplained encephalopathy, or an extreme reaction requiring hospitalisation to an otherwise supposed mild illness such as gastroenteritis. Some children may have unusual food behaviours such as protein aversion seen most commonly in the urea cycle disorders. A history of selfinjurious behaviour can be seen in Lesch-Nyhan syndrome and hyperammonaemia; other psychiatric abnormalities can be associated with disorders of cobalamin and later onset lysosomal storage disorders. The nature of the developmental delay should be recorded. Any suggestion of regression of skills makes an IEM much more likely and merits intensive investigation. The pattern of delay can sometimes be of help: hypotonia causing gross motor delay is seen in the organic acidurias, fatty acid oxidation disorders, (FAOs) and urea cycle defects; and speech delay is often the first sign of Sanfilippo syndrome (mucopolysaccharidosis type III). Generalised delay with associated sensori-motor hearing loss suggests a mitochondrial disorder. Seizures may occur in association with developmental delay. A severe seizure disorder in the newborn period occurs in non-ketotic hyperglycinaemia, peroxisomal disorders and in sulphite oxidase/molybdenum cofactor deficiency. Beyond infancy, epilepsy is quite common in mildly delayed children and would not specifically indicate an IEM. A severe seizure

\begin{tabular}{ll|}
\hline Table 2 Examination findings \\
\hline Feature & Disorder(s) \\
\hline Dysmorphism & $\begin{array}{l}\text { Smith-Lemli-Opitz, other cholesterol synthesis disorders } \\
\text { Congenital disorders of glycosylation } \\
\text { Peroxisomal disorders } \\
\text { Lysosomal disorders } \\
\text { Lysosomal storage disorders, glycogen storage disorders } \\
\text { Lysosomal storage disorders, fatty acid oxidation defects, mitochondrial }\end{array}$ \\
$\begin{array}{ll}\text { Cardiomyopathy } & \text { disorders } \\
\text { Smell } & \text { Organic acid disorders } \\
\text { Neurological signs } & \text { Mitochondrial disorders, organic acidurias, pterin defects } \\
\text { Dystonia } & \text { Canavan's disease, Tay-Sachs, L-2-hydroxyglutaric aciduria, glutaric } \\
\text { Macrocephaly } & \text { aciduria type I } \\
\text { Microcephaly } & \text { Sulphite oxidase deficiency, maternal PKU offspring, previous } \\
\text { hyperammonaemia, previous hypoglycaemia, GLUT-1 deficiency, neuronal } & \text { ceroid lipofuscinosis 1 } \\
\text { Many IEMs, e.g. organic acid, amino acid, urea cycle disorders }\end{array}$ \\
$\begin{array}{l}\text { Growth-failure to thrive, short } \\
\text { stature } \\
\text { Hair-coarse, "kinky" } \\
\text { Skin-coarse, ichthyosis, eczema }\end{array}$ & $\begin{array}{l}\text { Menkes disease, MPS disorders, arginino-succinic aciduria } \\
\text { LSD, Conradi-Hunermann, biotinidase deficiency, Sjogren-Larsson }\end{array}$ \\
\hline & \\
\hline
\end{tabular}


Table 3 Baseline investigations; global delay with no specific associated findings

\begin{tabular}{ll}
\hline Blood & Urine \\
\hline Full blood count & Organic acids \\
Urea, sodium, potassium, calcium & $\begin{array}{l}\text { Amino acids } \\
\text { Glycosaminoglycans }\end{array}$ \\
Liver function tests: ALT, ALP, rGT, albumin & \\
Lactate & \\
Creatine kinase & \\
Thyroid function tests (TSH, free thyroxine) & \\
Ammonia & \\
Urate & \\
Amino acids &
\end{tabular}

disorder and delay is seen in many of the lysosomal storage disorders, but in these cases there is usually neurological regression giving stronger indication of IEM. The newly described group of creatine deficiency disorders can cause seizures and developmental delay, although more usually there are extrapyramidal movements and hypotonia.

Examination findings can be very helpful (table 2). Dysmorphic features may lead to a syndromic diagnosis or suggest specific IEMs such as Smith-Lemli-Opitz syndrome. The presence of hepatosplenomegaly accompanying developmental delay is highly suggestive of a lysosomal storage disorder (LSD). Other features that may be present in LSDs are corneal clouding, facial coarsening, and dysostosis multiplex on skeletal survey. Cardiac muscle abnormalities such as hypertrophic or dilated cardiomyopathy are seen in FAOs where the developmental delay is usually in the gross motor domain due to hypotonia. A characteristic odour is associated with certain organic acidurias such as maple syrup urine disease (sweet) and isovaleric aciduria ("sweaty feet") but is rarely a leading sign. Specific neurological signs such as dystonia make one suspect an IEM. In particular, dystonia is frequently a manifestation of mitochondrial disease and organic acidurias after a metabolic crisis in which basal ganglia are damaged. Macrocephaly is seen in Canavan's disease, L-2-hydroxyglutaric aciduria, glutaric aciduria type I, and Tay-Sach's disease, while microcephaly is characteristic of sulphite oxidase deficiency but may be seen as a result of previous metabolic encephalopathy due, for example, to hyperammonaemia. The offspring of poorly controlled or unrecognised phenylketonuria (PKU) have microcephaly. This diagnosis is especially worth considering where the mother has emigrated to the UK from a country where universal newborn screening for PKU is not undertaken.

Despite all these possible clues, the most common situation challenging paediatricians is of the child with global developmental delay and no accompanying clues on history or examination. Investigation of these children gives a low yield for IEMs; previous data suggest that the yield after metabolic investigation in this group is $1-5 \%{ }^{1}$ It is proposed that a reasonable and safe approach therefore is to limit investigation to the battery of baseline tests in table 3 . Interpretation of these baseline tests requires consideration of their reliability and in particular factors which may cause spurious or misleading results. Some of these factors are discussed below.

Blood lactate can be a useful screen for disorders of energy metabolism. These include disorders of gluconeogenesis, disorders of pyruvate metabolism, and respiratory chain mitochondrial disorders. If there is associated hypoglycaemia then gluconeogenic disorders are more likely. Care is needed, however, in interpreting a high blood lactate. Excessive screaming, tourniquet pressure or haemolysis may cause an erroneously elevated lactate level, making mildly elevated lactate levels (up to $3 \mathrm{mmol} / \mathrm{l}$ ) difficult to interpret. Increased lactate accompanied by elevation of plasma alanine and proline suggests a chronic lactic acidaemia, thereby aiding intepretation. CSF lactate is a more reliable indicator of elevated lactate and can even be raised when plasma lactate is normal if the clinical presentation is largely neurological. Similar care is needed in interpreting blood ammonia levels as it may too be artificially elevated by the same circumstances as lactate, with the added problem of contamination. Elevated ammonia suggests inadequate function of the urea cycle. This may be due to a primary urea cycle defect or secondary to liver disease, drugs such as sodium valproate, or another inborn error. Urine organic acids should detect nearly all organic acid defects and some fatty acid oxidation defects. However it is important to be aware that some disorders may be difficult to detect with biochemical tests when the child is well as abnormalities in these circumstances may be minimal. Repeat testing during the illness is therefore important. Raised creatine kinase is a non-specific finding in fatty acid oxidation defects or other disorders affecting muscle. Urate measurement has been included as a low concentration may indicate a defect of purine metabolism or molybdenum cofactor deficiency and a high concentration is seen in several IEMs such as Lesch-Nyhan syndrome and glycogen storage disorders. Urinary glycosaminoglycans have been included in the first line screen since mucopolysaccharidosis type III (Sanfillipo disease) can present solely as developmental delay. ${ }^{5}$ Since the coarse facial dysmorphism typically associated with MPS disorders is generally absent in this condition, clinicians may not consider an MPS disorder as a cause of pure developmental delay.

These tests should diagnose or provide clues to many IEM including organic acid, amino acid, urea cycle defects, fat oxidation disorders, and mucopolysaccharidoses. Serial review in children with developmental delay is important. Time may reveal a new symptom such as the repetitive hand movements of Rett syndrome, or a protein aversion may become clearer as the child reaches an age where they would eat a more varied diet. Organomegaly becomes more obvious as the disorder progresses and slowly evolving regression is only obvious as time elapses. A suggested time frame for review of patients with developmental delay with no aetiology is two assessments in the first year of life, annually until school age, and once more at puberty. In the absence of any associated specific features, we do not recommend any additional metabolic investigations.

\section{Specific investigations for IEM}

In situations where developmental delay is associated with a specific finding - that is, hypotonia, regression, eye abnormalities, hepatosplenomegaly, or dysmorphism it is valuable to "tailor" investigations to those IEM which can present with these factors. Table 4 groups disorders with associated tests, under these headings. Before embarking on these investigations it may be appropriate to discuss the case with a specialist metabolic paediatrician. After such discussion it is likely that specialised investigations are initiated in parallel with (or even by-passing) the baseline tests.

The physician should consult the local specialist laboratory for specimen requirements as details may vary between laboratories. A metabolic assay directory is available for tests provided in the UK by the National Metabolic Biochemistry Network (www.metbio.net).

We suggest that following these guidelines makes "missing" an IEM extremely unlikely and forms a rational basis for the approach to investigating the child with developmental delay for the presence of IEM. The suggested staged approach minimises costs and limits the use of the most expensive tests to selected cases. 


\begin{tabular}{|c|c|}
\hline Disorder & Test \\
\hline \multicolumn{2}{|l|}{ Hypotonia } \\
\hline Peroxisomal disorders & Very long chain fatty acids (P) \\
\hline Biotinidase deficiency & Biotinidase assay $(P)$ \\
\hline \multirow[t]{2}{*}{ Purine/pyrimidines disorders } & Urate (B) and (U) \\
\hline & Purine/pyrimidines (U) \\
\hline Neurotransmitter deficiencies & Biopterins $(B)+(C)$ \\
\hline \multirow{2}{*}{$\begin{array}{l}\text { Fattly acid oxidation defects } \\
\text { Organic acid disorders }\end{array}$} & Acylcarnitines (P) \\
\hline & Organic acids (U) \\
\hline \multicolumn{2}{|l|}{ Neurological regression } \\
\hline \multirow[b]{2}{*}{$\begin{array}{l}\text { Biofinidase deficiency } \\
\text { Mucopolysaccharidoses }\end{array}$} & Biotinidase (P) \\
\hline & $\begin{array}{l}\text { Urine glycosaminoglycans (U) } \\
\text { White cell enzymes (B) }\end{array}$ \\
\hline \multirow{2}{*}{$\begin{array}{l}\text { Neuronal ceroid lipofuscinosis } \\
\text { Other lysosomal disorders }\end{array}$} & Vacuolated lymphocytes, NCL enzymes, mutations (B) \\
\hline & White cell enzymes (B) \\
\hline \multirow{3}{*}{ Mitochondrial disorders } & Lactate (B) and (C) \\
\hline & Respiratory chain enzymes (muscle and skin) \\
\hline & Mitochondrial mutations/deletions (B and muscle) \\
\hline \multirow{2}{*}{ Neurotransmitter deficiencies } & Neurotransmitters (C) \\
\hline & Biopterins (B) and (C) \\
\hline Organic acid disorders & $\begin{array}{l}\text { Organic acids (U) } \\
\text { Acylcarnitines (P) }\end{array}$ \\
\hline \multicolumn{2}{|l|}{ Eye signs } \\
\hline \multirow{2}{*}{$\begin{array}{l}\text { Peroxisomal disorders } \\
\text { Disorders of cholesterol synthesis }\end{array}$} & Very long chain fatty acids (P) \\
\hline & 7-dehydrocholesterol, cholesterol (B) \\
\hline Mitochondrial disorders & Lactate (B), CSF lactate \\
\hline Congenital disorders of glycosylation & Transferrin isoforms (B) \\
\hline \multirow{3}{*}{$\begin{array}{l}\text { Sulphite oxidase deficiency } \\
\text { Homocystinuria }\end{array}$} & Urate $(P)$, sulphites $(U)$ \\
\hline & Homocysteine (P) \\
\hline & Amino acids (homocystine) (U) \\
\hline \multirow[t]{2}{*}{ Lysosomal storage disorders } & Glycosaminoglycans (U) \\
\hline & White cell enzymes (B) \\
\hline Cerebrotendinous xanthomatosis & Bile acids (U) \\
\hline \multicolumn{2}{|l|}{ Severe seizure disorder } \\
\hline \multirow{2}{*}{$\begin{array}{l}\text { Sulphite oxidase deficiency/molybdenum cofactor } \\
\text { deficiency }\end{array}$} & Urate $(P)$ \\
\hline & Sulphites (U) \\
\hline \multirow{2}{*}{$\begin{array}{l}\text { Biotinidase deficiency } \\
\text { Neuronal ceroid lipofuscinosis }\end{array}$} & Biotinidase \\
\hline & Vacuolated lymphocytes, NCL enzymes, mutations (B) \\
\hline Non-ketotic hyperglycinaemia & Amino acids ( $P$ and $U$ ) \\
\hline Creatine deficiency & $\begin{array}{l}24 \text { hour creatine:creatinine, specific metabolites } \\
\text { MRS brain }\end{array}$ \\
\hline Hepato(spleno)megaly & \\
\hline Lysosomal storage disorders & Glycosaminoglycans (U) \\
\hline & White cell enzymes (B) \\
\hline Urea cycle disorders & $\begin{array}{l}\text { Aligosaccharides (U) } \\
\text { Ammonia }\end{array}$ \\
\hline & $\begin{array}{l}\text { Amino acids (P) and (U) } \\
\text { Orotic acid (U) }\end{array}$ \\
\hline Bile acid disorders & Bile acids (U) \\
\hline Glycogen storage disorders & Lactate (B), glucose profile, cholesterol, triglycerides \\
\hline Developmental delay plus dysmorphism & \\
\hline Smith-Lemli-Opitz & $\begin{array}{l}\text { 7-dehydrocholesterol, cholesterol (P) } \\
\text { Cholesterol precursors (P) }\end{array}$ \\
\hline Other cholesterol precursor disorders & \\
\hline Peroxisomal disorders & Very long chain fatty acids (P) \\
\hline & $\begin{array}{l}\text { Plasmalogens } \\
\text { (XR epiphyses) }\end{array}$ \\
\hline Congenital disorders of glycosylation & Transferrin isoforms (P) \\
\hline Mucopolysaccharide disorders and other storage & Glycosaminoglycans (U) \\
\hline disorders & White cell enzymes (B) \\
\hline
\end{tabular}

\section{SUMMARY}

While IEMs are not a common cause of developmental delay, their investigation remains very important for several reasons: appropriate genetic counselling can be given; specific therapies may be available; and clinicians can be aware of any associated risk of acute metabolic decompensation. When no diagnosis is made on first referral, there is value in review at infrequent intervals as new features may become apparent or new IEMs described. While it is almost impossible to assert that one has completely excluded an IEM, the investigative plan outlined above provides a sensible framework for testing and would detect the vast majority of IEMs that are associated with developmental delay.

\section{Authors' affiliations}

M A Cleary, Great Ormond Street Hospital NHS Trust, London, UK A Green, Birmingham Children's Hospital NHS Trust, Birmingham, UK Competing interests: none 


\section{REFERENCES}

1 Shevell MI, Ashwal S, Donley D, et al. Practice Parameter: Evaluation of the child with global developmental delay: Report of the quality standards subcommittee of the American Academy of Neurology and the Practice Committee of the Child Neurology Society. Neurology 2003;60:367-80.

2 Majnemer A, Shevell MI. Diagnostic yield of the neurologic assessment of the developmentally delayed child. J Pediatr 1995;127:193-9.

3 Curry CJ, Stevenson RE, Aughton D, et al. Evaluation of mental retardation: recommendations of a consensus conference:
American College of Medical Genetics. Am J Med Genet 1997;72:468-77.

4 Gringras $P$. Choice of medical investigations for developmental delay: a questionnaire survey. Child Care Health Dev 1998;24:267-76.

5 Cleary MA, Wraith JE. Management of mucopolysaccharidosis type III. Arch Dis Child 1993;69:403-6.

6 Zytkovicz TH, Fitzgerald EF, Marsden D, et al. Tandem mass spectrometric analysis for amino, organic and fatty acid disorders in newborn dried blood spots: a two-year summary from the New England Newborn Screening Program. Clin Chem 2001;46:1945-55.

\section{IMAGES IN PAEDIATRICS}

\section{Erythema induratum of Bazin and episcleritis in a 6 year old girl}

$\mathrm{A}$ 6 year old girl of Nigerian extract presented with a three month history of tender lesions on the backs of her legs. Some of these lesions had superficially ulcerated (fig 1). She also had a non-painful red right eye for approximately one month.

She was born in Nigeria, having arrived with her family in Ireland the previous year. She received the BCG vaccination in Nigeria. She was systemically well, afebrile, with normal growth parameters. Noone in her immediate family had any evidence of active tuberculosis.

She had a nodular episcleritis in her right eye which responded to beclomethasone drops. A 2TU tuberculin skin test performed on admission was very strongly positive with an area of induration in excess of $3 \mathrm{~cm}$ in diameter. This later blistered and ulcerated.

The skin lesions fitted the description of erythema induratum of Bazin (EIB), one of a group of cutaneous hypersensitivity reactions to internal tuberculosis infection known as tuberculids. This was confirmed by histological report on a punch skin biopsy which revealed the classical granulomatous dermatitis and lobular panniculitis (fig 2). No organisms were seen on staining, and polymerase chain reaction for tuberculosis was negative. A chest radiograph was normal.

EIB is uncommon in children, but cases have been reported even in infants. ${ }^{1}$
Unlike erythema nodosum, it occurs more often on the posterior aspect of the legs, can ulcerate, and typically has a more prolonged course. ${ }^{2}$ It necessitates a thorough investigation for tuberculosis and responds well to appropriate antituberculous medications.

T R Leahy

Department of Paediatrics, Mid-Western Regional Hospital, Limerick, Ireland

P Downey

Department of Pathology, Mater Misericordiae Hospital, Dublin, Ireland

B Ramsay

Department of Dermatology, Mid-Western Regional Hospital R K Philip

Department of Paediatrics, Mid-Western Regional Hospital

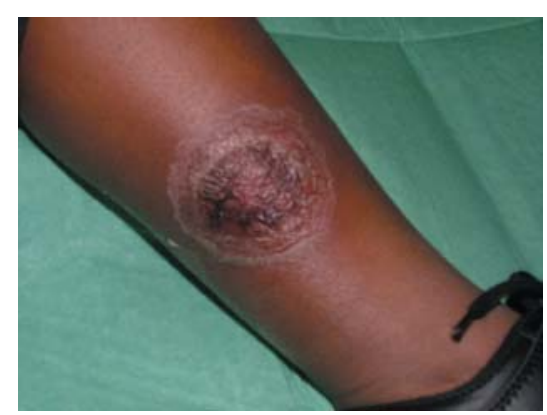

Figure 1 Area of shallow ulceration on medial aspect of left leg.
Correspondence to: R K Philip, Department of Paediatrics, Mid-Western Regional Hospital, Limerick, Ireland; roy.philip@mailh.hse.ie

Competing interests: none declared

Consent was obtained for publication of the figures

\section{References}

1 Chang MW, Lawrence R, Orlow SJ. Erythema induratum of Bazin in an infant. Pediatrics 1999; 103:498-9.

2 McNutt NS, Moreno A, Contreras F. Inflammatory disease of the subcutaneous fat. In: Lever's histopathology of the skin, 8th edn. Philadelphia, PA: Lippincott-Raven, 1997:429-40.

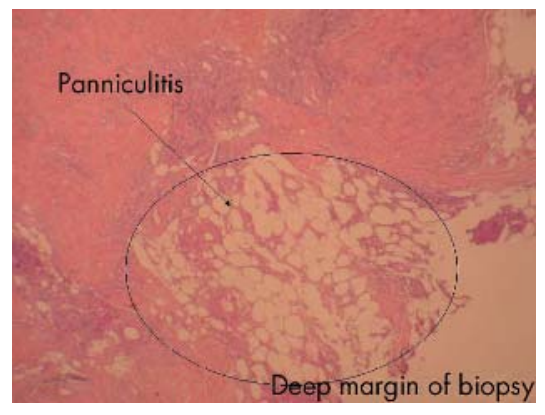

Figure 2 Skin biopsy from leg lesion with granulomatous inflammation and panniculitis $(H \& E, 100 \times)$. 